\title{
A CLINICAL STUDY ON HIV-TB CO-INFECTION
}

\author{
Sheela Mathew ${ }^{1}$, Sandeep ${ }^{2}$
}

${ }_{1}^{1}$ Additional Professor, Department of Infectious Diseases, Government Medical College Hospital, Kozhikode, Kerala, India. 2Senior Resident, Department of Medicine, Government Medical College Hospital, Kozhikode, Kerala, India.

\begin{abstract}
BACKGROUND ABSTRACT

Tuberculosis has been a major public health problem worldwide for centuries. It is one of the common opportunistic infections in HIV infected individuals and is well known that it spreads via respiratory tract, can be treated effectively and if proper precaution is taken even transmission can be prevented. HIV is driving the TB epidemic in many countries including India. In countries with high HIV prevalence, TB has high morbidity and mortality. Extrapulmonary and smear-negative pulmonary cases are more in number and at the same time difficult to diagnose. Also, drug toxicity is high, and outcome is poor, partly due to coexistence of other HIV related infections. The risk of recurrence is higher.

Aims- The study was designed to analyse the clinical profile and manifestation of HIV-TB co-infection and its relationship with CD4 count and to evaluate usefulness of various investigatory modalities in making a diagnosis, assess the response to treatment and to find out the incidence of MDR-TB.
\end{abstract}

\section{MATERIALS AND METHODS}

This was an observational study. A total of 101 patients diagnosed to have HIV-TB co-infection were selected for the study. The patients who were selected for the study were evaluated in detail clinically and with laboratory investigations. The investig ations were used as a part of minimal diagnostic work-up needed to confirm the diagnosis of HIV-TB co-infection and were individualised for every patient.

\section{RESULTS}

Major symptoms included weight loss, fever, anorexia, tiredness, cough, sputum, headache, night sweats, dyspnoea, haemoptysis and swelling. Out of 101 cases, 25 had isolated pulmonary involvement, 49 had both pulmonary and extrapulmonary involvement and 27 had extrapulmonary involvement. Isolated pulmonary involvement occurred in 25\% HIV-TB co-infection. Though significant weight loss was seen in HIV infection, accelerated weight loss of more than $10 \%$ in one-month favoured tuberculosis. Dissemination was common in HIV-TB co-infection and it occurred at low CD4 counts. There was no statistical correlation between the manifestation of Tuberculosis and the CD4 count. IRIS occurred in patients with low CD4 counts when started on HAART. Disseminated TB and miliary TB were observed below CD4 200. Imaging had an important role in the work-up of TB and some invasive procedures could be avoided because of it. 55\% patients had slow response to treatment (ATT and HAART). Anti-TB drug resistance was proved in 5\% cases and 3\% turned out to be MDR-TB.

\section{CONCLUSION}

Isolated pulmonary involvement occurred in 25\% HIV-TB co-infection. Extrapulmonary involvement occurred in around $75 \%$. There was no statistical correlation between the manifestation of Tuberculosis and the CD4 count. Dissemination is common in HIV-TB co-infection and it occurs at low CD4 counts. There was significant observation that disseminated TB and miliary TB were observed below CD4 200. Even if chest x-ray is normal, a sputum AFB and AFB culture is ideal if facilities are available. Moreover, drug resistant strains can be picked up in patients with poor response characteristics. Repeated smear for AFB should be done in patients started on empirical ATT and culture may be sent in those with strong clinical suspicion. Imaging, especially ultrasound in working up cases of PUO, in HIV and following up patients with HIV-TB co-infection is more rewarding in a resource limited setting than doing complex invasive investigations or waiting for other invasive procedures. Routine AFB stain may be done on smears of lymph node aspirates and histopathology slides, as this will identify more number of TB. Flare up of TB as IRIS may be anticipated in those patients with low CD4 was started on HAART. There was no significant correlation between low CD4 count and slow response to treatment. Further studies are needed to study the poor response characteristics including nutrition and immunity.

\section{KEY WORDS}

HIV-TB Co-Infection, IRIS, Extrapulmonary TB, Disseminated TB.

HOW TO CITE THIS ARTICLE: Mathew S, Sandeep. A clinical study on HIV-TB co-infection. J. Evolution Med. Dent. Sci. 2018;7(31): 3491-3495, DOI: $10.14260 / \mathrm{jemds} / 2018 / 786$

'Financial or Other Competing Interest': None.

Submission 28-05-2018, Peer Review 17-07-2018,

Acceptance 23-07-2018, Published 30-07-2018.

Corresponding Author:

Dr. Sheela Mathew,

Government Medical College Hospital,

Kozhikode, Kerala, India.

E-mail: shaarongardens@yahoo.com

DOI: $10.14260 /$ jemds $/ 2018 / 786$

(c) (i) $(-)$

\section{BACKGROUND}

Since the time of its initial description, HIV-AIDS has exponentially progressed all around the globe showing no sign of regression. Occurrence of HIV-AIDS pandemic has led to a dramatic increase in number of TB cases worldwide. Tuberculosis is a major public health problem worldwide for centuries. It is one of the common opportunistic infections in HIV infected individuals and is of particular importance because it is contagious by the respiratory route, readily treatable and potentially preventable. HIV is driving the TB 
epidemic in many countries including India. TB is the leading cause of morbidity and mortality in patients with HIV-AIDS. TB and HIV share a deadly synergism and increase the risk by 150 times for developing active TB. Individuals affected by Tubercle bacilli have a $10 \%$ lifetime risk of developing active disease. The risk increases $10 \%$ per year if they are coinfected with HIV.

There are many risk factors for TB, of which HIV infection stands first. PLWHA are more prone to develop progressive disease following primary $\mathrm{TB}$ and also there is increased chance of reactivation of latent TB. There is high chance of reinfection as well. The estimated annual risk of reactivation among those co-infected with HIV and TB is $5-8 \%$; cumulative lifetime risk is $30 \%$ or more. ${ }^{1}$ However, in HIV negative persons cumulative lifetime risk is $5-10 \%$. The course of HIV infection is accelerated subsequent to development of TB. Compared to CD4 count matched HIV infected controls without TB, the relative risk of death and development of other opportunistic infection is higher in HIVTB co-infected patients. ${ }^{2}$

HIV infection increases the demands on TB programmes, which are struggling to cope with the increased TB case load. The rise in TB suspects is putting a strain on diagnostic services. Extrapulmonary and smear-negative pulmonary TB cases, which are more difficult to diagnose account for an increased proportion of total cases. There are more adverse drug reactions. There is higher morbidity and mortality, partly due to other curable HIV related infections. The risk of TB recurrence is higher.

HIV infection is not a predisposing factor for MDR-TB. Many factors contribute for development of MDR-TB. This include increased susceptibility to TB, overcrowding, frequent hospital visits and exposure to MDR-TB cases, subtherapeutic blood levels due to malabsorption of drugs, poor drug compliance etc. increases chance of development of MDR-TB.

Active TB occurs at any CD4 count, whereas other opportunistic infections occur at CD4 count below 200. In patients with $>200 \mathrm{CD} 4$, pulmonary TB is more frequently seen. Chest $\mathrm{x}$-ray shows upper lobe infiltrates and cavitations similar to HIV negative individuals. Sputum AFB may be positive. As immunosuppression progresses, ЕРTB becomes increasingly common. In contrast to HIV negative persons with EPTB, the disease is often disseminated. With advanced disease x-ray may show lower lobe involvement, consolidation can be seen similar to bacterial pneumonia and cavity may be absent. Sputum smears are negative for AFB. ${ }^{3}$ Intrathoracic or mediastinal adenopathy is seen resembling primary $\mathrm{TB}$, even if the person has a past history of $\mathrm{TB}$ exposure. Miliary TB is also commonly seen associated with severe immunosuppression. A considerable proportion of patients (10 - 20\%) with advanced immunosuppression may have apparently normal looking chest x-ray, yet bacilli can be isolated from the sputum. However, CT thorax demonstrates abnormalities such as pulmonary nodules, tuberculomas and intrathoracic lymphadenopathy in these patients. Common forms of EPTB are pleural effusion, extra thoracic lymph node $\mathrm{TB}$, meningitis and abdominal TB.

Diagnosis of TB in HIV infected patients is often difficult due to several reasons. ${ }^{4}$ Negative sputum smears, atypical chest x-ray findings, higher prevalence of EPTB especially at sites which are inaccessible and resemblance to other opportunistic pulmonary infections, all may cause delay in diagnosis. Diagnostic approach to diagnose TB in HIV infected persons are similar to that in immune competent persons, but invasive diagnostic procedures are more often required to establish the diagnosis. The mortality of HIV infected patients with TB is comparatively higher than that of HIV negative TB patients. The mortality depends on the type of disease and the degree of underlying immunosuppression. In TB meningitis with co-infection mortality is about $60-70 \%$.

\section{Aims and Objectives}

1. To study the clinical manifestations of the spectrum of Tuberculosis in HIV infected individuals.

2. To find a correlation between CD4 count and the manifestations of Tuberculosis in HIV infected individuals.

3. To assess the usefulness of various investigatory modalities helping to make a diagnosis of HIV-TB coinfection.

4. To study the response of HIV-TB co-infection to ATT.

5. To find out the incidence of anti-TB drug resistance in HIV infected individuals.

\section{MATERIALS AND METHODS}

This was an observational study conducted in our institution. A total of 101 patients diagnosed to have HIV-TB co-infection and who were admitted in ward or attending ART OP were selected for the study. The patients who did not give an informed consent, those aged less than 12 years and who were not willing for anti-TB or anti-retroviral therapy were excluded. The patients who were selected for the study were evaluated in detail clinically and with laboratory investigations. Laboratory investigations included CBC, ESR, urine analysis, RFT, LFT, blood sugar etc. Diagnostic investigations included HIV ImmunoComb assay, CD4 + count, sputum AFB smear and culture, Tuberculin test, ultrasound, CT scan, MRI, pleural/ ascitic fluid study, lumbar puncture, FNAC, biopsy, echocardiography etc. The investigations were used as a part of minimal diagnostic workup needed to confirm the diagnosis of HIV-TB coinfection and were individualised for every patient. All patients enrolled in the study were followed up for minimum period of 6 months. Data was analysed by standard statistical techniques using Epi Info software. Correlation between CD4 and type of Tuberculosis is done using chi-square test.

\section{RESULTS}

A total of 101 cases were studied. Cases included 80 males and 21 females. The age varied from 17 - 61 with a mean age of 38 years. They were categorised into 5 groups.

\begin{tabular}{|c|c|}
\hline$<20$ & 1 \\
\hline $21-30$ & 16 \\
\hline $31-40$ & 46 \\
\hline $41-50$ & 30 \\
\hline$>50 \quad$ Age Distribution \\
\hline \multicolumn{2}{|c|}{} \\
\hline
\end{tabular}

\section{Major Symptoms}

Major symptoms included weight loss (101), fever (96), anorexia (94), tiredness (76), cough (75), sputum (48), headache (47), night sweats (30), dyspnoea (20), 
haemoptysis (19) and swelling (6). Out of 101 cases 25 had isolated pulmonary involvement, 49 had both pulmonary and extrapulmonary involvement and 27 had extrapulmonary involvement.

\section{CD4 Count}

93 out of 101 patients had a CD4 count at the time of study. Counts were categorised into 3 groups.

\begin{tabular}{|c|c|}
\hline CD4 Count & Frequency \\
\hline$<50$ & 17 \\
\hline $51-200$ & 49 \\
\hline$>200$ & 27 \\
\hline Total & 93 \\
\hline CD4 Count and Frequency \\
\hline
\end{tabular}

$66 \%$ had CD $4<200$ at the time of study. Lowest value was 2 and highest was 1133/uL. On further enquiry, detection of TB prompted screening for HIV in 56 patients. 44 patients had contact with a case of TB earlier. All the 21 females had their spouses infected with HIV. 13 of 21 females had their spouses with a recent history of treatment for TB.

18 of 101 patients had a past history of TB before detection of HIV. 6 patients had history suggestive of primary complex in childhood. The details were obtained by interviewing their parents or close relatives.

\begin{tabular}{|c|c|c|c|}
\hline Pattern of TB & CD4 < 50 & CD4 50-200 & CD4 > 200 \\
\hline Pulmonary & 8 & 7 & 13 \\
\hline PTB + EPTB & 7 & 24 & 14 \\
\hline EPTB & 2 & 8 & 4 \\
\hline Miliary & 0 & 3 & 0 \\
\hline Effusion & 0 & 6 & 3 \\
\hline \multicolumn{4}{|c|}{ Correlation of Pattern of TB with CD4 } \\
\hline
\end{tabular}

Single table analysis was done with Chi-squared test to get a correlation between CD4 and type of Tuberculosis. The p-value was 0.3971. 49 patients had combined pulmonary and extrapulmonary involvement, of which 30 were having disseminated infections evidenced by radiological, histological or microbiological involvement of liver, spleen and lymph nodes. 8 had hepatic involvement alone, 5 had hepatic and splenic involvement, 3 had lymph node involvement and 3 had radiological evidence of miliary TB. 27 patients had extrapulmonary involvement alone. 15 had disseminated TB and 12 had single organ EPTB.

\begin{tabular}{|c|c|}
\hline Disseminated TB & 15 \\
\hline Pericardial TB & 3 \\
\hline TB Peritonitis & 2 \\
\hline TB Meningitis with Tuberculomas & 3 \\
\hline Lymph Node TB & 3 \\
\hline Intestinal TB & 1 \\
\hline Total & $\mathbf{2 7}$ \\
\hline Types of Extrapulmonary $\mathbf{T B}$ \\
\hline
\end{tabular}

Types of Pulmonary Involvement

67 patients had involvement of lungs clinically and radiologically. 6 patients had clinical involvement without radiological shadow. 42 patients had sputum positivity for AFB, of which 6 had normal chest x-ray. Five patients, in spite of sputum negativity, yielded culture positivity. They had non-homogenous opacities on chest x-ray. Three patients had cavitary lesions clinically and radiologically. 2 of them were sputum positive at presentation and one developed positivity 2 months after treatment. 8 patients had pleural effusion, out of which 3 were positive for AFB. 53 had non-homogenous opacities on chest x-ray suggestive of active Tuberculosis, among them 34 were positive for AFB in sputum. All 3 miliary TB patients were sputum positive. The radiological picture was co-related with CD4 count. P-value was 0.6442 . The zone of radiological involvement was also co-related with CD4 (pvalue 0.4466 ).

\begin{tabular}{|c|c|c|c|}
\hline $\begin{array}{c}\text { Radiological } \\
\text { Finding }\end{array}$ & Total & $\begin{array}{c}\text { Sputum + for } \\
\text { AFB }\end{array}$ & $\begin{array}{c}\text { Sputum - for } \\
\text { AFB }\end{array}$ \\
\hline $\begin{array}{c}\text { Non-homogenous } \\
\text { opacities }\end{array}$ & 53 & 34 & 19 \\
\hline Cavitary lesion & 3 & 2 & 1 \\
\hline Miliary mottling & 3 & 3 & 0 \\
\hline Pleural effusion & 8 & 3 & 5 \\
\hline Chest X-Ray Findings in PuImonary Involvement and \\
Sputum Smear Results \\
\hline
\end{tabular}

\section{CNS Involvement}

Twelve out of 101 patients had Tuberculous Meningitis; 6 had Tuberculomas diagnosed by CT/MRI. MRI picked up 2 cases of Tuberculomas, which were negative by CT. Five patients presented with seizures; one with dementia and one with hemiplegia. Five patients were comatose at presentation. 6 had signs of meningeal irritation, 3 had false localising signs with $6^{\text {th }}$ cranial nerve palsy bilaterally, 1 patient developed obstructive hydrocephalus and succumbed to it. Only one patient had CD 4 count below 50 . Four had CD 4 above 200. Nine out of 12 had clinical features of dissemination. Nine had CSF study suggestive of TB meningitis. Two had very low TLC and elevated protein and hypoglycorrhachia.

\section{Pericardial Involvement}

Pericardium was involved as tuberculous pericardial effusion in 5 cases. All were confirmed by echocardiography along with aspiration of pericardial fluid. Two patients had pericardial effusion as only manifestation at presentation, but developed pulmonary and lymph node involvement subsequently. Two cases had evidence of dissemination. One case had miliary TB.

\section{Lymph Node Involvement}

Lymph nodes were clinically palpable in 47 patients. All had cervical lymph nodes, 16 had axillary nodes, 3 had para-aortic nodes, 3 had inguinal lymphadenopathy and 3 had epitrochlear involvement. While combining physical examination and an imaging modality (USG, CT, MRI), 63 had lymph node enlargement.

\section{Lymph Node Histology}

Lymph node histology was studied by FNA, AFB staining and those negative were subjected to excision biopsy. Out of 47 studied 10 had AFB positive, 25 revealed granulomas by aspiration or excision biopsy, 12 did not reveal granulomas or AFB, but treated because of clinical suspicion; 5 out of these during treatment with ART and ATT became AFB positive. 


\section{Abdominal Involvement}

USG and other imaging modalities were used to assess abdominal organ involvement. During USG, focal lesion in the liver and spleen and enlargement of para-aortic lymph nodes were given significance. 18 patients had focal lesions in liver, 12 had liver and spleen involvement and another 18 had liver, spleen and para-aortic node involvement. 4 patients had isolated para-aortic node involvement. 2 patients had intestinal TB. One was ileocaecal TB and other a mass in rectum which on biopsy was proved as TB. One patient presented as mass in epigastrium. Imaging showed pancreatic mass, responded very well to ATT. 8 patients had ascites. Skeletal TB was seen in one and genitourinary TB was diagnosed in another one patient. $87.8 \%$ of patients were anaemic and average $\mathrm{Hb}$ was $9.3 \mathrm{gm} \%$.

\section{Mantoux Test}

Mantoux test was done in 90 patients. 27 showed positivity ( $>5 \mathrm{~mm}$ ). Mantoux positivity was seen predominantly in patients with high CD4 counts $(>200)$, ( $p$ value $<0.05)$.

\begin{tabular}{|c|c|c|c|}
\hline Mantoux Test & CD4 < 50 & CD4 50 - 200 & CD4 > 200 \\
\hline Positive & $3(17 \%)$ & $12(22 \%)$ & $12(44 \%)$ \\
\hline Negative & 14 & 37 & 15 \\
\hline Total & $\mathbf{1 7}$ & $\mathbf{4 9}$ & $\mathbf{2 7}$ \\
\hline \multicolumn{2}{|c|}{ Tuberculin Response- Correlation with CD4 Count } \\
\hline
\end{tabular}

\section{Imaging}

Imaging plays an important role in diagnosis of HIV-TB coinfection. It was observed that some chest x-rays are normal in spite of severe symptoms and smear positivity. Six such cases were noted in the study. In the presence of radiographic lesion suggestive of $\mathrm{TB}$ with negative smears, the best approach is to give a course of broad-spectrum antibiotics and repeat imaging. Lack of improvement or deterioration would indicate TB. USG abdomen was crucial in the diagnosis of abdominal TB. Focal lesions in liver and spleen and lymph node enlargement could be picked up easily. CT of abdomen was taken in only few cases, in whom findings of ultrasound was inconclusive. CT head was useful in tuberculomas and TB meningitis and also for diagnosing hydrocephalus. MRI was more sensitive in picking up tuberculoma.

\section{AFB Staining}

Sputum was sent in all patients for AFB staining. 42 patients had sputum positivity. Staining of lymph node aspirate for AFB was more productive than routine cytology staining. Yield of AFB from CSF and pleural fluid smears were nil.

\section{Follow-Up}

Treatment of patients was started according to WHO/ NACO guidelines. 4 cases succumbed to illness before starting any treatment. Time taken for improvement was assessed by interviewing for subjective well-being and improvement in general condition along with investigational parameters. 32\% of patients improved within 15 days of starting ATT. 53.6\% patients took more than two months to improve. This included patients with IRIS who worsened in the initial days of therapy, those with disseminated TB and lower CD4 count. However, there was no significant correlation between CD4 and time of improvement.

\section{ATT AND MDR-TB}

Twenty samples were subjected to culture and 9 came out as positive. 5 of them were subjected to sensitivity and found resistance in one or more drugs. 3 cases were detected as MDR-TB. No cases of XDR-TB were seen.

\section{DISCUSSION}

Out of 101 cases, 80 were males (79\%) and 21 were females. This reflects the higher incidence of HIV infection in males in this part of the country. Moreover, the number of males coming up for treatment is more compared to females. To bring out females more into the treatment options will require more women empowerment.

The average age of patients was 38.3 yrs. The maximum number of patients were in the 31 - 40 age group. This shows that the most productive years of life are lost due to the disease. CD4 count ranged from 2 to 1133. Unlike other opportunistic infections which occur below CD4 200, Tuberculosis can occur at any CD4 count. But majority of patients were having CD4 between $50-200.55 \%$ of patients were diagnosed to have HIV, while they presented with tuberculosis in the present study. The risk of developing tuberculosis in HIV infected individuals is $10-15 \%$ per annum compared to lifetime risk of 5 - 10\% in HIV negative individuals. Routine screening for HIV has been included as a part of RNTCP program in India. The tuberculosis clinics therefore form an important entry point for HIV diagnosis, care and support. Co-ordination and cross talk between these two government programs, thus improve the outcome of patients with HIV-TB and also control the burden of Tuberculosis in India. 44 of 101 patients reported contact with TB at least once in their lifetime. In a country like India where Tuberculosis is endemic, history of contact is insignificant. Only 6 patients had primary complex treated.

Severe weight loss is a presenting feature of HIV infected patients infected with TB unlike other OIs. So weight loss points towards Tuberculosis in HIV infected persons and the index of suspicion should be high. Hence, investigational workup can be directed towards Tuberculosis in a resource limited setting like that we have in India. Among other significant symptoms, night sweats deserve a special mention. Even though not much significance is given to this symptom in contemporary literature, night sweats in a patient with HIV-TB co-infection raise index of suspicion for dissemination or extrapulmonary involvement. Out of 101 cases $25 \%$ had isolated pulmonary TB, $48 \%$ had combined pulmonary and extrapulmonary involvement including 3 miliary TB and $27 \%$ had extrapulmonary involvement alone. This finding is similar to many studies on HIV-TB coinfection. Types of tuberculosis were correlated with the CD4 count at presentation using chi-squared test with single table analysis. P-value was 0.3971 . This was not statistically significant. Pulmonary Tuberculosis were more in the CD4>200 group compared with other OIs. As the study was based on IP admissions due to Tuberculosis and patients attending the ART clinic, the number of patients with CD4 count > 200 were less and many cases of Pulmonary Tuberculosis occurring at higher CD4 count might have been missed. This might have influenced the $\mathrm{p}$-value to make it insignificant. Extrapulmonary with pulmonary involvement including dissemination was more in patients with CD4 < 200 , indicating the immunosuppression in this group. 
Lack of sputum positivity in 50\% patients suggests immune suppression. This point to the fact that if clinical suspicion is strong all possible modalities of investigation like culture, molecular methods, imaging etc. may be used for prompt diagnosis. The radiological picture was co-related with CD4 count. P-value was 0.6442. Though it is not statistically significant, it was observed that miliary shadows were seen predominantly in patients with CD4 count $<200$. Non-homogenous opacities were seen in all CD4 ranges. Generally, the involvement of lower zone increases with decrease of CD4 count. The zone of radiological involvement was also co-related with CD4. P-value was 0.4466 which is statistically insignificant, but it is seen that diffuse involvement of all zones was seen predominantly in patients with CD4 count $<200$. In this study, $75 \%$ of patients had extrapulmonary involvement. This is like many other studies. Similarly like many other studies, lymph node was the commonest extrapulmonary site affected. Despite the high prevalence of pulmonary and extrapulmonary TB in Indian patients, TB meningitis was less common when compared to cryptococcal meningitis. In this study, $12 \%$ had TB meningitis. 6 patients had tuberculomas. Only 6 patients had signs of meningeal irritation. It is well described that classical signs may be absent in HIV associated meningitis. Mantoux positivity was seen predominantly in patients with high CD4 counts ( $>200)$ and this observation was statistically significant. ( $\mathrm{P}$ value $<0.05$ ). Previous studies have reported $58 \%$ energy for tuberculin response in co-infected patients with CD4 $<200$. Though the percentage positivity is low, positive predictive value of Mantoux test increases as the CD4 decreases. ${ }^{5}$ Swaminathan et al reported a positive predictive value as low as $18.8 \%$ when CD 4 was > 200 and $48.3 \%$ if CD4 is $<200 .^{5}$ Out of 101 cases, 14 had IRIS (14\%). Marfatya et al reported incidence of $6.2 \% .6$ Two patients succumbed to IRIS. This shows that starting HAART along with ATT is a definitive risk factor for IRIS.

As described in literature granulomas on lymph node histopathology was not seen in all cases. $53 \%$ of lymph node studied revealed granulomas. FNAC was very useful in demonstrating acid fast bacillus. ZN staining of lymph node aspirate could pick up AFB in a greater number of cases. $26 \%$ (12 samples) of lymph node studied did not reveal granulomas or AFB. But when started on ATT, 5 became positive on ZN staining. This shows extent of immune suppression in these patients and the improvement in immunity once started treatment. Few cases showed increase in size of lymph nodes and suppuration on starting ATT. This may be a part of IRIS. It was impossible to use clinical judgment alone to diagnose abdominal involvement. Most cases were picked up by imaging as part of work-up for PUO. USG was handy for demonstrating focal lesions in liver and spleen. Patients were started on ATT and looked for response. Focal lesions in liver and spleen, which responded to ATT were considered as tuberculosis. In a resource limited setting USG is very helpful and start treatment to look for a response, if no response further invasive investigations can be carried out.

\section{CONCLUSION}

Isolated pulmonary involvement occurred in 25\% HIV-TB coinfection. Extrapulmonary involvement occurred in around $75 \%$. Though significant weight loss is seen in HIV infection, accelerated weight loss of $>10 \%$ body weight in one-month favours tuberculosis. There was no statistical correlation between the manifestation of Tuberculosis and the CD4 count. Dissemination is common in HIV-TB co-infection and it occurs at low CD 4 counts. Incidence of disseminated TB in the present study is $42 \%$. There was significant observation that disseminated TB and miliary TB were observed below CD4 200. Even if chest x-ray is normal, a sputum AFB and AFB culture is ideal if facilities are available. Moreover, drug resistant strains can be picked up in patients with poor response characteristics. Repeated smear for AFB should be done in patients started on empirical ATT and culture sent in those with strong clinical suspicion. Imaging especially ultrasound in working up cases of PUO in HIV and following up patients with HIV-TB co-infection is more rewarding in a resource limited setting than doing complex invasive investigation or waiting for other invasive procedures. Routine AFB stain may be done on smears of lymph node aspirates and histopathology slides as this will identify a greater number of TB. Flare-up of TB as IRIS may be anticipated in those patients with low CD4 started on HAART. $55 \%$ patients had slow response to treatment (ATT and HAART). There was no significant correlation between low CD4 count and slow response to treatment. Further studies are needed to study the poor response characteristics including nutrition and immunity. Anti-TB drug resistance was proved in $5 \%$ cases and $3 \%$ turned out to be MDR-TB. The percentage was low because culture was done only in $20 \%$ patients.

\section{REFERENCES}

[1] Narain JP, Raviglione MC, Kochi A. HIV associated tuberculosis in developing countries: epidemiology and strategies or prevention. Tuber Lung Dis 1992;73(6):311-21.

[2] Sharma SK, Mohan A, Kadhiravan T. HIV-TB coinfection: epidemiology, diagnosis \& management. Indian J Med Res 2005;121(4):550-67.

[3] Vanham G, Edmonds K, Qing L, et al. Generalised immune activation in pulmonary tuberculosis: coactivation with HIV infection. Clin Exp Immunol 1996;103(1):30-4.

[4] Tripathy S, Narain JP, Sharma SK, et al. Tuberculosis and HIV infection. Chap- 36. In: Sharma SK, ed. Tuberculosis. Jaypee 2007:404-12.

[5] Swaminathan S, Deivanayagam CN, Rajasekharan S, et al. Long term follow up of HIV infected patients with tuberculosis treated with 6 months intermittent short course chemotherapy. Natl Med J Indian 2008;21(1):38.

[6] Marfatiya YS, Ghiya R. HIV -TB confection: a study of 246 cases. Proceedings of HIV Congress 2008:55-6. 